\title{
Hábito alimentar do tatu-canastra (Xenarthra, Dasypodidae) em uma área de cerrado do Brasil Central
}

\author{
Teresa Cristina S. Anacleto ${ }^{1}$ \\ Jader Marinho-Filho ${ }^{2}$
}

\begin{abstract}
Food habits of the giant armadillo (Xenarthra, Dasypodidae) in an area of grassland of central Brasil. The diet of the giant armadillo - Priodontes maximus (Kerr, 1792) - was studied in the field at Fazenda São Miguel, Unai, Minas Gerais, Brazil, through 82 samples collected at foraging excavations and 25 fecal samples. In both types of sample the most common item was insects (Isoptera e Hymenoptera) and, in less quantity, plant fragments and others invertebrates (Aranae, Blattaria, Coleoptera, Diplopoda, Scorpiones). These data suggest the giant armadillo is a specialist on insects with an opportunistic foraging strategy.

KEY WORDS. Priodontes maximus, giant armadillo, food habits, insectivore, grassland, Brazil
\end{abstract}

O tatu-canastra, Priodontes maximus (Kerr, 1792) Xenarthra: Dasypodidae, ocorre em grande parte da América do Sul, a leste dos Andes, desde a Venezuela, Colômbia e Guianas até a Argentina, Paraguai e Brasil (WETzeL 1982). No Brasil não existem registros na região nordeste (WETZEL 1985) e no leste brasileiro esta espécie é cada vez mais rara (SILVA 1984). Goiás, Mato Grosso e a região do Gran Chaco foram consideradas áreas de maior densidade populacional (CABRERA \& YEPES 1940). O tatu-canastra tem ampla distribuição geográfica e pode ocupar diferentes hábitats, desde florestas de terras baixas e altas até savanas, mas as áreas abertas são o hábitat preferencial (WETZEL 1982). Fatores como a expansão da agropecuária e a forte pressão de caça representam os mais sérios riscos para a espécie.

O tatu-canastra foi classificado como vulnerável pela União Internacional para Conservação da Natureza (IUCN) e está no Apêndice 1 do CITES Comércio Internacional das Espécies da Flora e da Fauna Silvestres em Perigo de Extinção (NowaK 1991). O Instituto Brasileiro do Meio Ambiente e dos Recursos Naturais Renováveis (IBAMA), o incluiu na Lista Oficial da Fauna Brasileira Ameaçada de Extinção (BERNARDES et al. 1990). BARRETO et al. (1985) estudaram o conteúdo estomacal de três espécimes e os principais itens encontrados foram cupins e formigas. É a espécie mais mirmecófaga (REDFORD 1985) entre os Dasypodidae e suas adaptações incluem a grande habilidade em

1) Departamento de Ciências Biológicas, Universidade do Estado de Mato Grosso. Caixa Postal 08, 78690-000 Nova Xavantina, Mato Grosso, Brasil.

E-mail: teresacristinaa@yahoo.com

2) Departamento de Zoologia, Universidade de Brasília. 70910-900 Brasília, Distrito Federal, Brasil. 
escavar, a língua vermiforme, grandes glândulas salivares, porção posterior da mandíbula reduzida, dentes pequenos e em número variável (PATTERSON 1975), aproximadamente 100 (IHERING 1968; NowAK 1991), que caem de acordo com a idade dos indivíduos (EISENBERG 1989; NOWAK 1991).

Devido a inexistência de trabalhos sobre o tatu-canastra, o presente trabalho teve como objetivo investigar a dieta dessa espécie, numa área de Cerrado do Brasil Central, a partir da análise de organismos presentes nos fossados e nas fezes.

\section{MATERIAL E MÉTODOS}

O presente trabalho foi realizado numa área natural (10.000 ha) da Fazenda

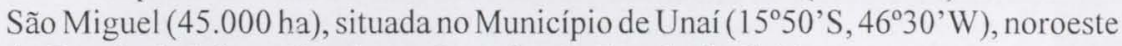
do Estado de Minas Gerais, na fronteira do Estado de Goiás.

A área de estudo reúne os principais tipos fisionômicos do Cerrado com predomínio de cerrado stricto sensu (83\%) associado com campo limpo (14\%), mata e vereda (2\%) (FUNATURA 1994) e campo de murunduns (1\%). O clima caracteriza-se pela existência nítida de duas estações: uma chuvosa e quente (outubro a abril) e outra mais fria e seca (maio a setembro). A média anual de chuva é $112 \mathrm{~mm}$, com temperatura média anual máxima e mínima de 28 e $15^{\circ} \mathrm{C}$, respectivamente (19851995, Estação Meteorológica da Fazenda São Miguel).

As coletas foram realizadas no período de maio a novembro de 1997.

\section{Organismos coletados nos fossados}

Pela manhã eram localizados rastros e pegadas deixados nas trilhas. Estas evidências conduziam às escavações (tocas e fossados) do tatu-canastra feitas durante à noite. Os organismos presentes no interior e/ou sobre a terra escavada dos fossados eram coletados e fixados em álcool $70 \%$. Nos fossados feitos na base das palmeiras eram coletados os organismos e uma folha da palmeira para identificação. O mesmo procedimento de coleta foi repetido na palmeira mais próxima à planta fuçada, para verificar a preferência por determinada palmeira e os organismos relacionados a ela. Os substratos foram classificados nas seguintes categorias: cupinzeiros, formigueiros e solo. Este último foi considerado toda vez que não havia indícios aparente de cupinzeiro ou formigueiro, tais como galerias ou grânulos fecais.

Para cada item (categoria de presa) identificado foi calculada a freqüência de ocorrência, em função do número total de amostras. A associação de fossados com espécies de palmeiras foi analisada através do teste de Kolmogorov-Smirnov, a fim de verificar a representação diferencial entre os organismos identificados nas palmeiras fuçadas e nas não fuçadas. Foi aplicado o mesmo teste para os fossados nas palmeiras e os efetuados nos demais substratos. Nos testes foram considerados apenas os Isoptera e Hymenoptera, por apresentarem maior freqüência de ocorrência.

\section{Coleta e análise das fezes}

As amostras foram coletadas principalmente sobre a terra escavada que fica na entrada da toca. No ato da coleta foi registrado o hábitat (tipo fisionômico), o substrato (solo, cupinzeiro ou formigueiro) e o local de deposição (solo, fossado ou toca). As amostras, após secagem ao sol, foram acondicionadas em sacos de papel. 
No laboratório, as fezes permaneceram na água por 24 horas antes da lavagem em duas peneiras acopladas, com malhas de $0,49 \mathrm{~mm}$ e $0,15 \mathrm{~mm}$. O material retido foi examinado em microscópio estereoscópio e identificado no nível taxonômico de ordem, família, gênero e, quando possivel, espécie. A freqüência de ocorrência de cada item encontrado nas fezes foi calculada em função do número total de amostras.

A representação diferencial entre os organismos identificados nas amostras procedentes das fezes e dos fossados foi analisada através do teste de KolmogorovSmirnov. Nesta análise foram excluídos os itens de origem vegetal pela ausência destes nas amostras de fossados.

\section{RESULTADOS}

\section{Organismos coletados nos fossados}

Nas amostras dos fossados $(n=65)$ foram identificadas seis ordens, contendo principalmente Isoptera e Hymenoptera (Tab. I). Cada amostra apresentou em média 1,6 itens. Entre os Isoptera os gêneros mais freqüentes foram: Syntermes spp. (43,1\%), Nasutitermes spp. (21,5\%), Velocitermes spp. (20,0\%), Armitermes spp. $(6,2 \%)$, Cornitermes spp. $(4,6 \%)$, Labiotermes spp. $(3,1 \%)$ e Heterotermes spp. $(3,1 \%)$. Na ordem Hymenoptera os gêneros mais freqüentes foram: Camponotus spp. (9,2\%), Pheidole spp. (6,2\%), Conomyrma spp. (3,1\%) e Atta spp. (3,1\%). Em apenas duas amostras não foram registrados Isoptera e Hymenoptera. Em raras ocasiões o tatu-canastra destruiu completamente o termiteiro. Este fato pode estar associado à redução do número de cupins na porção epígea do termiteiro, levando-o a forragear em outro local.

As amostras de palmeiras, Palmae $(\mathrm{n}=35)$, pertenciam a cinco espécies diferentes: Allagoptera leucocalyx (Drude) O. Kuntze, Syagrus comosa (Mart.) Mart., S. petraea (Mart.) Becc, S. campestris (Mart.) H. Wendl e Butia eriospatha (Mart.) Becc. Apenas nas três primeiras havia fossados. Tanto nas palmeiras fuçadas $(\mathrm{n}=17)$ quanto em suas vizinhas mais próximas não fuçadas $(\mathrm{n}=18)$, Allagoptera leucocalyx foi a mais freqüente com 10 exemplares $(58 \%)$ fossados e sete não fossados (3\%). Nas 35 amostras de palmeiras foram identificadas nove ordens de insetos: Aranae, Blattaria, Coleoptera, Hymenoptera, Isoptera, Scorpiones, Diptera, Hemiptera e Neuroptera. Houve predomínio de Isoptera, com os gêneros Nasutitermes spp. (23\%), Velocitermes spp. (17\%) e Diversitermes diversimiles Silvestri, 1901 (14\%). Na ordem Hymenoptera, a segunda mais freqüente, predominou a subfamília Myrmicinae (11\%). Os fossados foram efetuados nas palmeiras, independentemente da espécie, isto é, a proporção das espécies fuçadas não diferiu significativamente das não fuçadas (KS Dmax $=0,40 ; p=0,82$ ). Não houve diferença significativa entre as ordens Isoptera e Hymenoptera encontradas nas palmeiras fuçadas e não fuçadas (KS Dmax $=0,33 ; p=0,66$ ). As freqüências de Isoptera e Hymenoptera em fossados efetuados nas palmeiras não diferiram dos outros fossados, efetuados nos demais substratos (KS Dmax $=0,22 ; p=0,96)$.

Dos 82 fossados recentes, onde foram coletados os organismos, grande parte $(88 \%)$ foi escavada no cerrado. Cupinzeiro foi o substrato mais escavado, com 30 fossados, seguido por solo e formigueiro com 27 e 25 fossados, respectivamente. 
Tabela I. Freqüência (\%) de itens identificados em 65 amostras de fossados de tatu-canastra (Priodontes maximus), coletadas na Fazenda São Miguel, Unai, Minas Gerais, Brasil.

\begin{tabular}{|c|c|c|}
\hline Itens por familia & Número de amostras ( $\left(^{*}\right)$ & Porcentagem (\%) \\
\hline \multicolumn{3}{|l|}{ Aranae } \\
\hline Oonopidae & 1 & 1,5 \\
\hline Salticidae & 1 & 1,5 \\
\hline \multicolumn{3}{|l|}{ Blattaria } \\
\hline Não identificado & 1 & 1,5 \\
\hline \multicolumn{3}{|l|}{ Coleoptera } \\
\hline Cicindelidae & 1 & 1,5 \\
\hline \multicolumn{3}{|l|}{ Diplopoda } \\
\hline Não identificado & 1 & 1,5 \\
\hline \multicolumn{3}{|l|}{ Hymenoptera } \\
\hline \multicolumn{3}{|l|}{ Formicidae } \\
\hline Dolichoderinae & 2 & 3,1 \\
\hline Formicinae & 7 & 10,7 \\
\hline Myrmicinae & 12 & 18,3 \\
\hline Ponerinae & 2 & 3,1 \\
\hline \multicolumn{3}{|l|}{ Isoptera } \\
\hline \multicolumn{3}{|l|}{ Rhinotermitidae } \\
\hline Heterotermitinae & 2 & 3,1 \\
\hline \multicolumn{3}{|l|}{ Termitidae } \\
\hline Nasutitermitinae & 45 & 68,8 \\
\hline Termitinae & 4 & 6,1 \\
\hline
\end{tabular}

$\left({ }^{\star}\right)$ Número de amostras contendo o item em um total de 65 amostras.

\section{Análise das fezes}

Das 25 amostras de fezes, duas estavam na vereda, próximo à área de transição com o cerrado, onde o solo é seco e as demais estavam no cerrado. Quanto aos substratos, foram encontradas 17 amostras em formigueiros e oito no solo. A maioria $(72 \%)$ foi depositada na entrada das tocas ou sobre a terra escavada, o restante estava nos fossados (16\%) ou na trilha do animal (12\%). Cada amostra de fezes $(n=25)$ apresentou 6,1 itens, contendo principalmente Isoptera e Hymenoptera (Tab. II). Os gêneros mais freqüentes foram: Cornitermes spp. (68\%), Nasutitermes spp. (32\%), Armitermes spp. (20\%), Constrictotermes cyphergaster Silvestri, 1901 (20\%), Syntermes spp. (20\%), Diversitermes diversimiles (8\%) e Velocitermes spp. $(8 \%)$. Havia grande quantidade de partículas de solo, resíduos vegetais e partes esclerotizadas de invertebrados. Entre os itens vegetais foram identificadas folhas de Gramíneas (12\%), sementes de Annonaceae (Annona spp. 4\%) e ovos de dípteros $(56 \%)$. A integridade dos ovos indica que não passaram pelo trato digestivo do tatu-canastra. Provavelmente, os dípteros utilizam as fezes como recurso alimentar.

\section{Comparação entre fezes e fossados}

$\mathrm{Na}$ comparação dos itens identificados nas amostras de fezes e de fossados, consideramos apenas os itens de origem animal devido a ausência de vegetais nas amostras de fossado. Não houve diferença significa entre as freqüências de Isoptera 
e Hymenoptera presentes nas amostras de fezes e fossados (KS Dmax $=0,55 ; \mathrm{p}=$ $0,11)$. Nas amostras, tanto de fezes como de fossados, os Isopteras mais frequentes pertencem à subfamília Nasutitermitinae, sendo Syntermes spp. nos fossados e Cornitermes spp. nas fezes. Os Hymenoptera mais freqüentes pertencem às subfamílias Formicinae (Camponotus spp.) nos fossados e Ecitoninae (não identificado) nas fezes.

Tabela II. Freqüência (\%) de itens identificados em 25 amostras de fezes de tatu-canastra (Priodontes maximus), coletadas na Fazenda São Miguel, Unai, Minas Gerais, Brasil.

\begin{tabular}{|c|c|c|}
\hline Itens & Número de amostras $\left({ }^{\star}\right)$ & Porcentagem (\%) \\
\hline \multicolumn{3}{|l|}{ Invertebrados } \\
\hline Não identificado & 8 & 32 \\
\hline \multicolumn{3}{|l|}{ Vegetal } \\
\hline Folha & 3 & 12 \\
\hline Semente & 7 & 28 \\
\hline Pericarpo & 1 & 4 \\
\hline Não identificado & 25 & 100 \\
\hline \multicolumn{3}{|l|}{ Hymenoptera } \\
\hline \multicolumn{3}{|l|}{ Formicidae } \\
\hline Ecitoninae & 9 & 36 \\
\hline Formicinae & 7 & 28 \\
\hline Myrmicinae & 7 & 28 \\
\hline Não identificado & 7 & 28 \\
\hline \multicolumn{3}{|l|}{ Isoptera } \\
\hline \multicolumn{3}{|l|}{ Rhinotermitidae } \\
\hline Heterotermitinae & 3 & 12 \\
\hline \multicolumn{3}{|l|}{ Termitidae } \\
\hline Nasutitermitinae & 24 & 96 \\
\hline Termitinae & 3 & 12 \\
\hline \multicolumn{3}{|l|}{ Sobre as fezes } \\
\hline Acarina & 1 & 4 \\
\hline Diptera & 14 & 56 \\
\hline
\end{tabular}

$\left.{ }^{*}\right)$ Número de amostras contendo o item em um total de 25 amostras.

\section{DISCUSSÃO}

O tatu-canastra forrageou preferencialmente no cerrado, escavando no solo e em cupinzeiros. Seu olfato aguçado provavelmente detecta a fauna edáfica, como os cupins que transitam por galerias subterrâneas, levando-o a escavar no solo.

Os termiteiros proporcionam grande variedade de presas. Em um cupinzeiro podem coabitar até 14 espécies de cupins (DominGos et al. 1986), além de formigas, lacraias, opiliões, aranhas e escorpiões (MILL 1983). Essas associações podem explicar a diversidade de itens encontrados nas amostras fecais. No entanto, as ordens Aranae, Blattaria, Coleoptera e Diplopoda foram registradas apenas nos fossados. Isto pode estar relacionado com a disponibilidade da presa, a baixa ingestão ou a diferença na digestibilidade, inviabilizando a identificação durante a análise das fezes. 
A freqüência de ocorrência dos Termitidae nos fossados e nas fezes reflete a abundância desta família, que compreende cerca de $85 \%$ das espécies conhecidas no Brasil (CONSTANTINo 1999). Os cupins são a forma dominante de vida animal no Brasil central, tanto em biomassa (NEGRET \& REDFORD 1982) como em número de espécies, com cerca de 200 espécies descritas (MILL 1982), predominando a subfamília Nasutitermitinae (MiLL 1983; Domingos et al. 1986).

Syntermes spp. foram as presas mais freqüente nos fossados e Cornitermes spp. nas fezes. Provavelmente o comportamento, a acessibilidade e o inquilinismo das presas expliquem essa diferença. As espécies, como Syntermes spp., que evitam exposição direta durante $o$ ataque ao termiteiro, estão menos susceptíveis às investidas do predador. Os soldados surgem posteriormente para reconstrução das células quebradas. Termiteiros de Syntermes spp. são subterrâneos e em raras ocasiões possuem parte epígea, enquanto os de Cornitermes spp. são evidentes (DOMINGOS et al. 1986) e, em seus termiteiros velhos, pode viver mais de 10 espécies de inquilinos, (CONSTANTINO 1999), aumentando a oferta de alimento.

A dureza do termiteiro e as estratégias de defesa parecem não significar obstáculos ao tatu-canastra. O termiteiro de Cornitermes spp., que têm defesa química e mandibular, é 60 vezes mais duro que o de Nasutitermes spp. (NEGRET \& REDFORD 1982) que têm defesa química. Para atingir a parte hipógea do termiteiro de Cornitermes spp., o tatu-canastra escava o solo perpendicularmente ao termiteiro. Outra presa frequente foram as formigas de correição (Ecitoninae) que são agressivas e atacam com mordidas e picadas (REDFORD 1987; HÖLLDOBLER \& WILSON 1990; SUDD \& FRANKS 1987).

A habilidade na obtenção do alimento revelou-se através do consumo de Constrictotermes cyphergaster, uma espécie arborícola. Para fossar termiteiros distantes até $80 \mathrm{~cm}$ do solo, o tatu-canastra se mantém ereto, apoiado nos membros posteriores e na cauda, posição conspícua da ordem (WETZEL 1982).

A escolha das presas não se dá pelo tamanho. Os Syntermes spp. são grandes $(15,57 \mathrm{~mm})$, e as presas mais consumidas foram Cornitermes spp. $(9,55 \mathrm{~mm})$ e Nasutitermes spp. (4,32mm) (NEGRETT \& REDFORD 1982). Estudos indicam que a biomassa aumenta e a abundância decresce com o tamanho do corpo (BASSET \& KITHING 1991; STORK \& BLACKBURN 1993; MORSE et al. 1988). Provavelmente a abundância da presa interfira na escolha do tatu-canastra.

Quanto aos Hymenoptera, Camponotus spp. foram freqüentes nas fezes e nos fossados. Esta categoria de presa é mundialmente abundante (REDFORD 1987). Apenas na região neotropical ocorrem 248 espécies (MARICONI 1970). Algumas espécies desse gênero são agressivas, mas outras respondem a perturbações de seus ninhos com limitada resistência ou retração (HOLLDOBLER \& WILSON 1990).

As palmeiras apresentaram itens diferentes dos disponiveis nos demais substratos. Provavelmente são procuradas pela oferta de artrópodos a elas associados. O forrageamento ótimo prediz que a dieta fica mais generalizada quando o recurso alimentar torna-se escasso (EMLE 1966; MACARTHUR \& PIANKA 1966; SCHOENER 1971; CODY 1974). A presença de fragmentos vegetais em todas as 
amostras fecais do tatu-canastra sugere a existência de uma relação entre a composição da dieta e o recurso disponível. Como as amostras foram coletadas na seca, quando ocorre um acentuado declínio na abundância da entomofauna do Cerrado (DIETZ 1984), o tatu-canastra poderia estar substituindo os animais por vegetais. Outro item alimentar que corrobora esta sugestão são as formigas de correição (Ecitoninae), as mais freqüentes nas amostras fecais. Essas presas apresentam uma fase nômade quando uma grande colônia, composta por até um milhão de indivíduos, migra durante à noite (RETTENMEER et al. 1982).

A ingestão de partículas de solo provavelmente é acidental. O método de alimentação dos mirmecófagos não seleciona o alimento (REDFORD 1987). Este item pode auxiliar na suplementação das exigências minerais, minimizar distúrbios digestivos, devido ao efeito anti-ácido, e promover a absorção de toxinas (DAVIES \& BAILIE 1988).

Os dados indicam que o tatu-canastra é um insetívoro-especialista por consumir basicamente Isoptera e Hymenoptera e a composição da dieta varia de acordo com a disponibilidade das presas preferenciais no ambiente

\begin{abstract}
AGRADECIMENTOS. Ao Dr. Guarino R. Colli (Universidade de Brasilia) pelo auxílio na análise dos dados e Dr. Cláudio V. Pádua pelas valiosas sugestões. Às pessoas que auxiliaram na identificação do material e em especial ao Prof. Kiniti Kitayama (UnB), Prof. Paulo C. Motta (UnB) e Profa. Helena Castanheira (UnB). Aos funcionários da Fazenda São Miguel e ao Sr. Oswaldo Torres pela ajuda nos trabalhos de campo. A Fazenda São Miguel e ao Conselho Nacional de Desenvolvimento Científico e Tecnológico - CNPq pelo apoio financeiro.
\end{abstract}

\title{
REFERÊNCIAS BIBLIOGRÁFICAS
}

Barreto, M.; P. Barreto \& A. D'Alessandro. 1985. Colombian armadillos: stomach contents and infection with Tripanosoma cruzi. Jour. Mammal. 66: 188-193.

BASSET, Y. \& R.L. KITCHING. 1991. Species number, species abundance and body length of arboreal arthropods associated with an Australian rainforest tree. Ecol. Entomol. 16: 391-400.

BERnADES, A.T., A.B.M. MACHADO \& A.B. RYLANDS. 1990. Fauna brasileira ameaçada de extinção. Belo Horizonte, Fundação Biodiversitas para a Conservação da Diversidade Biológica, 62p.

CABrera A. \& J. Yepes. 1940. Mamiferos sud-americanos. Buenos Aires, Compañia Argentina de Editores, 370p.

Codv, M. L. 1974. Optimization in ecology. Science 183: 1156-1164.

Constantino, R. 1999. Chave ilustrada para identificação dos gêneros de Cupins (Insecta:Isoptera) que ocorrem no Brasil. Papéis Avuls Zool., São Paulo, 40 (25): 387-448.

Davies, A.G. \& I.C. Baillie. 1988. Soil eating by red leaf-monkeys (Presbytis rubicunda) in Sabah, northern Borneo. Biotropica 20 (3): 252-258.

DiETz, J.M. 1984. Ecology and social organization of the maned wolf (Chrysocyon brachyurus). Washington, Smithsonian Inst. Press, 51p.

Domingos, D.J., T.M.C.M. Cavenaghi; T.A. Gontijo; M.A. Drumond \& R.C.F. Carvalho. 1986. Composição em espécies, densidade e aspectos biológicos da fauna de térmitas de cerrado em Sete Lagoas-MG. Ciência Cultura 38 (1): 199-207.

EISENBERG, J.F. 1989. Mammals of the neotropics. London, Univ. Chicago Press, I+449p.

EMLE, J.M. 1966. The role of time and energy in food preference. Amer. Nat. 100: 611-617.

Funatura. 1994. Plano de manejo Santuário de Vida Silvestre São Miguel. Brasilia, Fundação Pró-Natureza, 82p. 
Hölldobler B. \& E.O. Wilson. 1990. The ants. Cambridge, Belknap Harvard Univ. Press, 732p. IHERING, R.V. 1968. Dicionário de animais do Brasil. São Paulo, Ed. Univ. Brasilia, 780p.

MacArthur, R.H. \& E.R. PianKa. 1966. On optimal use of a patchy environment. Amer. Nat. 110: 401-413.

Mariconi, F.A.M. 1970. As saúvas. São Paulo, Ed. Agronômica Ceres, 167p.

MiLl, A.E. 1982. Populações de térmitas (Insecta: Isoptera) em quatro hábitats no baixo rio Negro. Acta Amazônica 12 (1): 53-60.

. 1983. Generic keys to the soldier caste of new world termitidae (Isoptera:Insecta). Syst. Entomol. 8: 179-190.

Morse, D.R.; N.E. STORK \& J.H. LAWTON. 1988. Species number, species abundance and body length relationships of arboreal beetles in Bornean Iowland rain forest trees. Ecol. Entomol. 13: 25-37.

NEGRET, H.R.C. \& K.H. ReDFoRD. 1982. The biology of nine termites species (Isoptera: Termitidae) from the cerrado of central Brazil. Psyche 89: 81-106.

NOWAK, R.M. 1991. Walker's mammals of the world 2. Baltimore, Jonhs Hopkins Univ. Press, 1362p. Patterson, B. 1975. The fossil aardvarks (Mammalia: Tubulidentata). Bull. Mus. Comp. Zool. 147 (5): 185-237.

REDFORD, K.H. 1985. Foods habits of armadillos (Xenarthra: Dasypodidae), p. 429-437. In: G.G. MONTGOMERY (Ed.). The Evolution and ecology of sloths, armadillos, and vermilinguas. Washington, D.C., Smithsonian Inst. Press, 451 p.

. 1987. Ants and termites as food - patterns of mammalian myrmecophagy. Current Mammal. 1: 349-400.

Rettenmeyer, C.W.; R. Chadab-Crept; M.G. Naumann \& L. Morales. 1982. Comparative foraging by neotropical army ants, p. 59-73. In: P. JAISOON (Ed). Social insects in the tropics. Paris, Univ. Paris, 280p.

SCHOENER, T.W. 1971. Theory of feeding strategies. Ann. Rev. Ecol. Syst 11: 369-404.

SiLVA, F. 1984. Mamíferos Silvestres do Rio Grande do Sul. Porto Alegre, Fund. Zoobotânica do Rio Grande do Sul, 246p.

STORK, N.E.; T.M. BLACKBURN. 1993. Abundance, body size and bimass of arthropods in tropical forest. Oikos 67: 483-489.

SudD, J.H.; N.R. FranKs. 1987. The Behavioural ecology of ants. New York, Chapman \& Hall, 206p. WETZEL, R.M. 1982. Sistematics, distribution, ecology and conservation of South American edentates, p. 345-375. In: M.A. Mares \& H. Genoways (Eds). Mammalian biology in South America. Pennsylvania, Univ. Pittsburg, 539p.

1985. The taxonomy and distribution of armadillos, Dasypodidae, p. 23-46. In: G.G. MONTGOMERY (Ed.). The Evolution and ecology of sloths, armadillos, and vermilinguas. Washington, D.C., Smithsonian Inst. Press, 451p.

Recebido em 14.XI.2000; aceito em 12.VII.2001. 